\title{
Laparoscopic partial nephrectomy: a challenging operation with a steep learning curve
}

\author{
Anil Kapoor, MD, FRCSC
}

See related article on page 111

I n this issue of CUAJ, Tse and colleagues ${ }^{1}$ present an interesting study on the Regina experience with laparoscopic partial nephrectomy (LPN) for T1 renal tumours. The authors describe a single-institution experience of 47 cases of LPN with the introduction of an LPN utilization rate parameter, from a centre with a high volume of laparoscopic urological cases. Of this experience, 31 lesions resected were nonhilar tumours and 16 tumours were in hilar locations. Twenty-five percent of tumours resected were T1b tumours, with a maximum tumour size of $7.2 \mathrm{~cm}$. Eighty percent of renal lesions resected were malignant tumours. The mean tumour size was $3.8 \mathrm{~cm}$, and the mean operating time was 2.8 hours. The average hospital stay was 5 days, which seems somewhat higher than expected for laparoscopic procedures.

Operative hilar clamping in this experience was with en bloc clamping. This is in contrast to many centres, such as St. Joseph's Hospital, which preferentially clamps the renal artery only. This may decrease the intraoperative blood loss, and allows some renal perfusion. En bloc hilar clamping has the advantage that the individual renal artery and vein do not need to be dissected out, and may decrease the risk of injury to the major vessels during the procedure. It has become fairly standard that LPN requires some clamping of the renal hilum (either en bloc or renal artery only) to minimize intraoperative blood loss. This can be done with laparoscopic locking Satinsky clamps (Teleflex), locking DeBakey clamps or detachable laparoscopic bulldog clamps.

The authors discuss their experience with complications from LPN, which are not insignificant. The reported blood transfusion rate is $13 \%$ (6 patients), 1 patient required emergent postoperative nephrectomy for bleeding, 1 patient developed prolonged urinary leakage and 1 patient developed late calyceal stricture. Two patients had positive surgical margins (which likely will not compromise cancer control in the long term). These complications attest to the steep learning curve of this operation. Of almost 200 cases of LPN at St. Joseph's Hospital, the blood transfusion rate is not insignificant at $6 \%$, with 4 cases of postoperative pseudoaneurysm requiring radiological embolization. These postoperative issues are echoed by other high-volume centres. Meticulous suturing of transected blood vessels and collecting system reconstruction with parenchymal repair is required, all within an optimal warm ischemic clamp time of 30 minutes. In our experience, use of the absorbable Lapra-Ty (Ethicon) suture allows quick suturing to minimize ischemic time. Some centres use the permanent Hem-O-Lok (Pilling Weck) for parenchymal suturing to minimize ischemic time. Use of either Tisseel (Baxter) or Floseal (Baxter) may improve intraoperative hemostasis after parenchymal repair.

Is LPN a procedure that all urologists should be doing, or is this a procedure that should be done only in highvolume centres? The learning curve for this operation is steep, with the optimal number of cases likely in the 10-20 range. Urologists should select patients with nonhilar T1a lesions for their initial experiences with LPN. Overall, LPN is a difficult procedure to master, and the Regina experience is useful to disseminate to Canadian urologists.

Associate Professor of Surgery (Urology) and Chair, GU Oncology Program, Juravinski Cancer Centre, McMaster University, Hamilton, Ont.

Competing interests: None declared.

\section{Reference}

1. Tse A, Knaus R, Tse E. Laparoscopic partial nephrectomy: the University of Saskatchewan (Regina division) experience. Can Urol Assoc J 2009;3:111-8.

Correspondence: Dr. Anil Kapoor, McMaster Institute of Urology, St. Joseph's Healthcare, 50 Charlton Ave. E, Hamilton ON L8N 4A6; kapoor4@mamaster.ca 